\title{
MicroRNA-181 inhibits glioma cell proliferation by targeting cyclin B1
}

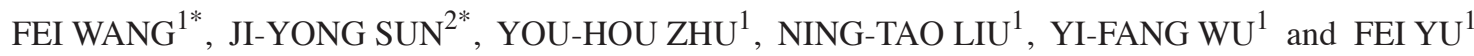 \\ ${ }^{1}$ Department of Neurosurgery, Tongji Hospital, Tongji University, Shanghai 200065; ${ }^{2}$ Department of Neurosurgery, \\ Wuxi Second Hospital Affiliated to Nanjing Medical University, Wuxi, Jiangsu 214002, P.R. China
}

Received October 15, 2013; Accepted May 2, 2014

DOI: $10.3892 / \mathrm{mmr} .2014 .2423$

\begin{abstract}
Small non-coding RNAs from the microRNA family (miRs) are important elements in the posttranscriptional control of gene expression. miRs are known to regulate numerous cellular processes and are of crucial importance during development and in pathological conditions, including tumor initiation and progression. In the present study, the expression level of miR-181 was reduced in glioma tissues compared with the adjacent normal tissues. The enforced expression of miR-181 was able to inhibit cell proliferation in U251 and SHG-44 cells, while antisense miR-181 oligonucleotides (antisense miR-181) enhanced cell proliferation. At the molecular level, these results further revealed that the expression of cyclin B1, a positive cell-cycle regulator, was negatively regulated by $\mathrm{miR}-181$. Therefore, the data reported in the present study demonstrates that miR-181 is an important regulator in glioma. These results may contribute to improving the understanding of the key misregulated miRNAs in glioma.
\end{abstract}

\section{Introduction}

Glioma is the most aggressive type of adult brain cancer $(1,2)$. Despite research efforts, the average lifespan for glioma patients postdiagnosis is $\sim 15$ months, with the majority of patients experiencing tumor relapse and outgrowth within seven months of initial radiation therapy $(3,4)$. Therefore, it is important to understanding the pathological mechanisms for tumor initiation and progression.

MicroRNA (miR) is a small non-coding RNA molecule, which functions in the transcriptional and posttranscriptional regulation of gene expression. Several recent studies have demonstrated that miRs have a critical role in cell

Correspondence to: Dr Fei Wang, Department of Neurosurgery, Tongji Hospital, Tongji University, 389 Xincun Road, Shanghai 200065, P.R. China

E-mail: fei_wangtj@126.com

*Contributed equally

Key words: microRNA-181, cyclin B1, glioma, cell proliferation proliferation, apoptosis and metastasis. Accumulating evidence has demonstrated the involvement of microRNAs in cancerous processes as either oncogenes or tumor suppressor genes $(5,6)$. Early investigations demonstrated that miR-181 downregulated the homeobox protein Hox-A11, a repressor of the differentiation process, which revealed the existence of a functional correlation between miR-181 and mammalian skeletal-muscle differentiation (7). Subsequently, it was identified that the expression levels of miR-181 were inversely correlated with Tcll oncogene expression in B-cell chronic lymphocytic leukemia samples (8). Furthermore, miR-181 was reported to function as a tumor suppressor, which triggered growth inhibition, induced apoptosis and inhibited invasion in multiple tumor types, including breast, colon and hepatocellular carcinoma (9-12). However, whether miR-181 is involved in the development of glioma remains largely unknown. Thus, the present study aimed to investigate the role of miR-181 in glioma cell proliferation.

\section{Materials and methods}

Cell culture and tissue samples. Glioma cells (U251 and SHG-44) were obtained from the American Type Culture Collection (Rockville, MD, USA). The cells were cultured in Dulbecco's modified Eagle's medium (Sigma, St. Louis, MO, USA) supplemented with $10 \%$ fetal bovine serum. The cultures were maintained at $37^{\circ} \mathrm{C}$ in a humidified atmosphere with $5 \% \mathrm{CO}_{2}$. The tumor tissues and adjacent normal non-tumor tissues were collected from routine therapeutic surgery at the Department of Thoracic Surgery, Provincial Hospital Affiliated to Shandong University (Jinan, China). All of the samples were obtained with informed consent and the present study was approved by the Ethics Committee of the Provincial Hospital Affiliated to Shandong University.

Analysis of miRNA expression using TaqMan reverse transcription polymerase chain reaction (RT-PCR). Total RNA from tissue samples and the cell lines was harvested using the miRNA Isolation kit (Ambion, Austin, TX, USA). The expression of mature miRNAs was assayed using a Taqman MicroRNA assay (Applied Biosystems, Shanghai, China) specific for hsa-miR-181. Briefly, 10 ng of total RNA were reverse transcribed to cDNA with the following specific stem-loop RT primers: Forward, 5'-UGGAAGGACGGGAAGUGGAA-3' 
and reverse, 5'-CCAGUGCAGGGUCCGAGGUA-3'. Quantitative (q)PCR was performed using an Applied Biosystems 7900 Real-time PCR system and a TaqMan Universal PCR Master mix (Applied Biosystems). All of the primers were obtained from the TaqMan miRNA assays. Small nuclear U6 snRNA (Applied Biosystems) was used as an internal control.

Plasmid construction and transfection. For the miR-181 expression plasmid, the human miR-181 precursor was cloned into pSilencer 4.1 (Ambion). The negative control plasmid consisted of a scrambled sequence (Ambion). To inhibit miR-181 function, an Ambion miRNA inhibitor for miR-181 was used, along with the negative control. For transfection, a complex of Lipofectamine ${ }^{\circledR} 2000$ (Invitrogen Life Technologies, Carlsbad, CA, USA) and $25 \mathrm{nM}$ miRNA mentioned above was prepared according to the manufacturer's instructions.

BrdU assays. A cell proliferation enzyme-linked immunosorbent assay (BrdU kit; Beyotime, Nantong, China) was used to analyze the incorporation of BrdU during DNA synthesis according to the manufacturer's instructions. All of the experiments were performed in triplicate. Absorbance was measured at $450 \mathrm{~nm}$ in the Spectra Max 190 ELISA reader (Molecular Devices, Sunnyvale, CA, USA)

Western blotting. The cells or tissues were harvested and lysed with ice-cold lysis buffer $(50 \mathrm{mM}$ Tris-HCl, $\mathrm{pH} 6.8$; $100 \mathrm{mM} 2-\mathrm{ME}, 2 \% \mathrm{w} / \mathrm{v}$ SDS and $10 \%$ glycerol). Following centrifugation at $20,000 \mathrm{x}$ g for $10 \mathrm{~min}$ at $4^{\circ} \mathrm{C}$, the proteins in the supernatants were quantified and separated by $10 \%$ SDS PAGE, and transferred onto a nitrocellulose membrane (Amersham Bioscience, Buckinghamshire, UK). Following blocking with $10 \%$ non-fat milk in PBS, the membranes were immunoblotted with cyclin B1 (Santa Cruz Biotechnology, Inc., Santa Cruz, CA, USA) and GAPDH (Abcam, Cambridge, MA, USA) antibodies, followed by anti-rabbit horseradish peroxidase-linked secondary antibodies (Cell Signaling Technology, Inc., Beverly, MA, USA). The signals were detected by a SuperSignal West Pico Chemiluminescent Substrate kit (Pierce Biotechnology, Inc., Rockford, IL, USA) according to manufacturer's instructions. Anti-cyclin B1 antibodies were purchased from Cell Signaling Technology, Inc. The protein levels were normalized to total GAPDH, using a mouse anti-GAPDH antibody (Santa Cruz Biotechnology, Inc., Santa Cruz, CA, USA).

Luciferase reporter assay. Total cDNA from the U251 cells was used to amplify the $3^{\prime}$ untranslated region (UTR) of cyclin B1 by PCR. The cyclin B1 3'UTR was cloned into pMir-Report (Ambion), yielding pMir-Report-cyclin B1. Mutations were introduced in potential miR-181 binding sites using the QuikChange site-directed mutagenesis kit (Stratagene, La Jolla, CA, USA). The cells were transfected with the 3'-UTR luciferase reporter and the miR-181 precursor plasmids for $36 \mathrm{~h}$. The pRL-SV40 vector (Promega Corporation, Madison, WI, USA) carrying the Renilla luciferase gene was used as an internal control to normalize the transfection efficiency. The luciferase values were determined using the Dual-Luciferase Reporter Assay system (Promega Corporation).

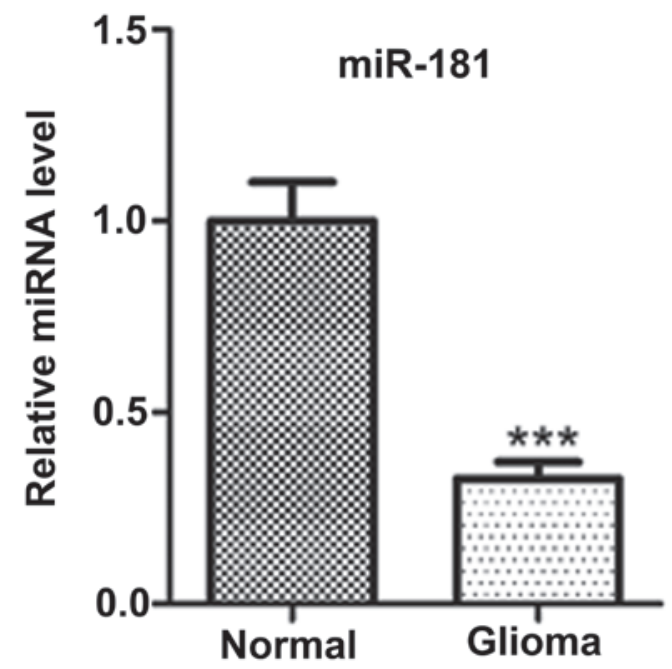

Figure 1. Expression levels of miR-181 in glioma tissues. miR-181 expression was determined by TaqMan quantitative polymerase chain reaction in human glioma tissues and adjacent non-cancerous tissues (normal). ${ }^{* * *} \mathrm{P}<0.001$, compared with normal tissues. miRNA, microRNA.

Statistical analysis. Data are expressed as the mean \pm standard error of the mean from at least three separate experiments. The differences between the groups were analyzed using Student's $\mathrm{t}$-test. $\mathrm{P}<0.05$ was considered to indicate a statistically significant difference.

\section{Results}

miR-181 expression levels are downregulated in patients with glioma. Firstly, to examine whether the miR-181 is differentially expressed in human glioma, its expression level was determined using TaqMan qPCR in 30 pairs of human glioma tissues and pair-matched adjacent non-cancerous tissues. The results demonstrated that the expression level of miR-181 was significantly decreased in glioma tissues compared with the adjacent non-cancerous tissues (Fig. 1).

miR-181 overexpression inhibits cell proliferation. In order to assess the effects of miR-181 on glioma cell growth, the miR-181 precursor was transfected into the U251 and SHG-44 cells, and cell growth post-transfection was examined. The miR-181 precursor was found to upregulate miR-181 expression (Fig. 2A and B), significantly reduce the cell number and inhibit the proliferation of cells post-transfection (Fig. 2C-F).

Inhibition of miR-181 promotes the proliferation of glioma cells. As described above, miR-181 has a critical role in the proliferation of glioma cells. However, whether inhibiting miR-181 enhances cell proliferation is unclear. Therefore, the two cell lines were transfected with antisense miR-181 and it was revealed that the ectopic expression of antisense hsa-miR-181 promoted the growth of U251 and SHG-44 cells, compared with that of the NC-transfected cells (Fig. 3A-D).

miR-181 directly targets cyclin B1 in glioma cells. Using a stringent bioinformatics approach (miRWalk software, Heidelberg, Germany; http://www.umm.uni-heidelberg. 
A

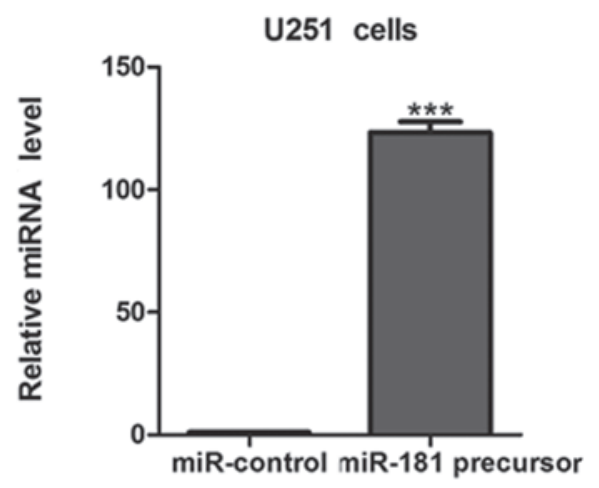

C

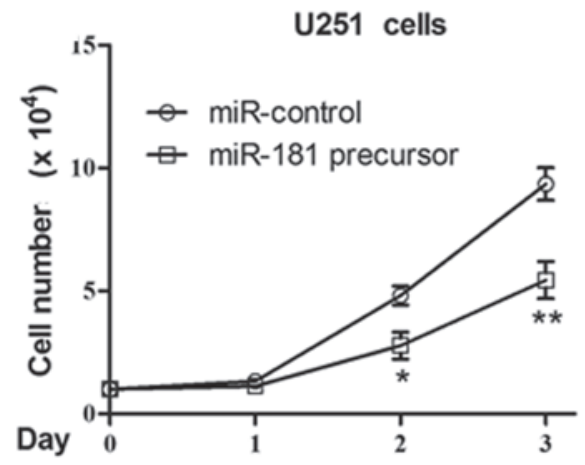

E

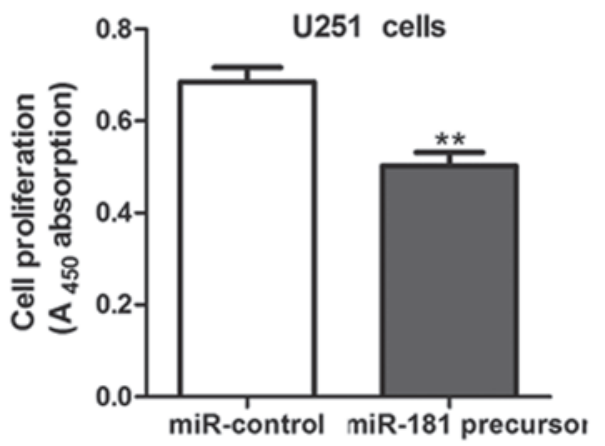

B

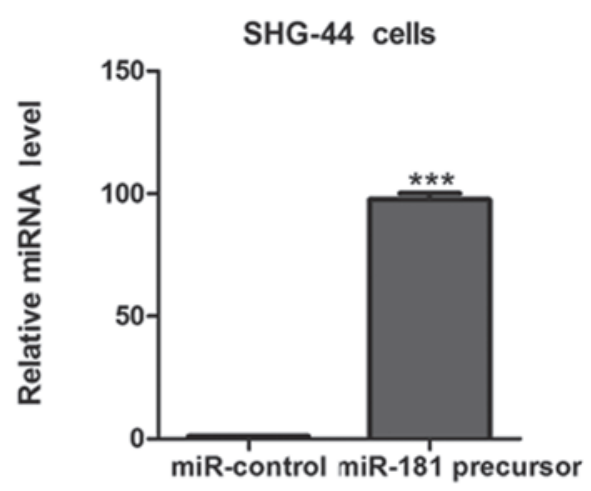

D

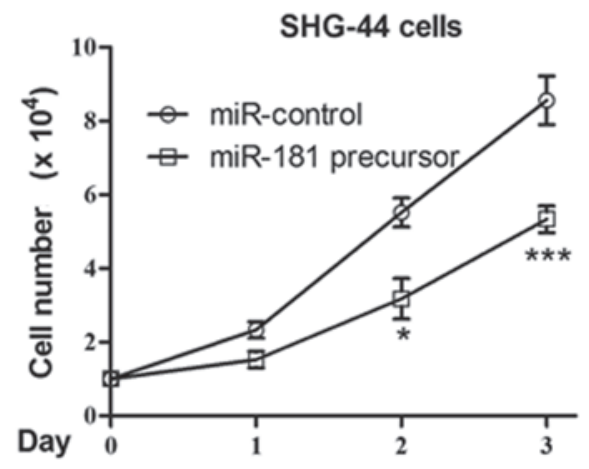

F

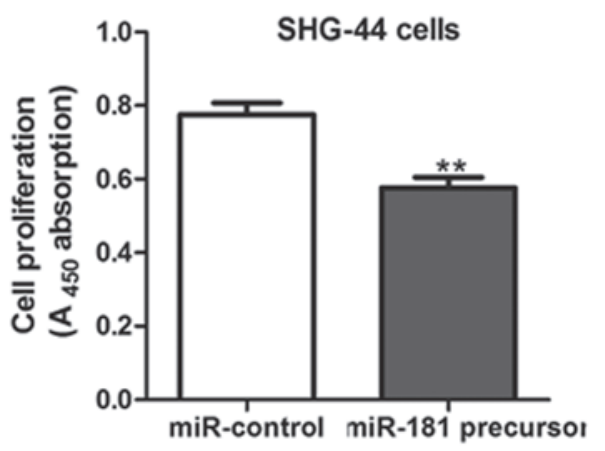

Figure 2. Overexpression of miR-181 inhibits glioma cell proliferation. (A-B) Expression of miR-181 was determined in U251 and SHG-44 cells following miR-181 precursor or negative control (miR-control) co-transfection, compared with the controls. The growth curve of (C) U251 and (D) SHG-44 cells following miR-181 precursor transfection, compared with the controls. Cell proliferative potential (BrdU) was determined in (E) U251 and (F) SHG-44 cells transfected with miR-181 precursor or negative control (Ctrl). A450 absorption was assayed following transfection for $24 \mathrm{~h}$. ${ }^{*} \mathrm{P}<0.05,{ }^{* * *} \mathrm{P}<0.01$ and ${ }^{* * * *} \mathrm{P}<0.001$, compared with the miR-control.

de/apps/zmf/mirwalk/), 24 putative human miR-181 target genes were identified (data not shown), among which the gene encoding cyclin B1 harbored a potential miR-181 binding site (Fig. 4A). The overexpression of miR-181 led to a reduction of luciferase activity when the reporter construct contained the cyclin B1 3'UTR (Fig. 4B). By contrast, mutations in the conserved miR-181 binding motif abrogated the reduced luciferase expression (Fig. 4B). Furthermore, the overexpression of miR-181 in glioma cells led to reduced cyclin B1 protein expression (Fig. 4C-D). Consistently, the inhibition of miR-181 led to an increased expression of cyclin B1 (Fig. 4E-F), further indicating that cyclin B1 is a target of miR-181 in glioma cells.

\section{Discussion}

In the present study, it was demonstrated that miR-181 expression is downregulated in glioma tissues. To the best of our knowledge, the present study was the first to identify at a molecular level that miR-181 regulated cyclin B1 expression by targeting its $3^{\prime}$ UTR. Collectively, these findings suggest that the downregulation of miR-181 may promote the initiation and progression of glioma. Notably, a recent study demonstrated that transiently overexpressed miR-181 significantly sensitized malignant glioma cells to radiation treatment, which was concurrent with the downregulation of B cell lymphoma/leukemia-2 (Bcl-2) protein expression (13). This indicates that miR-181 may modulate radiosensitivity by targeting Bcl-2 in human malignant glioma cells (13), suggesting that miR-181 may be a target for enhancing the effect of radiation treatment on malignant glioma cells. Therefore, the precise roles of miR-181 may be diverse in glioma cells.

It has been reported that several miRNAs were misregulated in glioma tissues or cells $(14,15)$. For example, miR-92b 
A

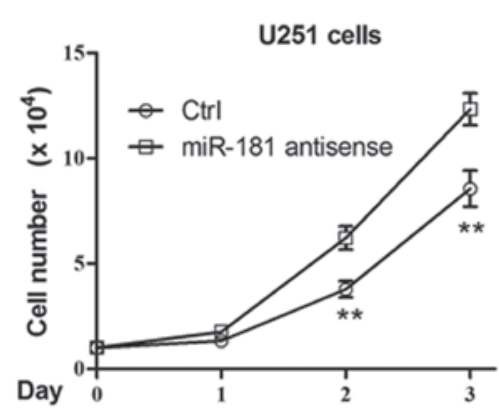

C

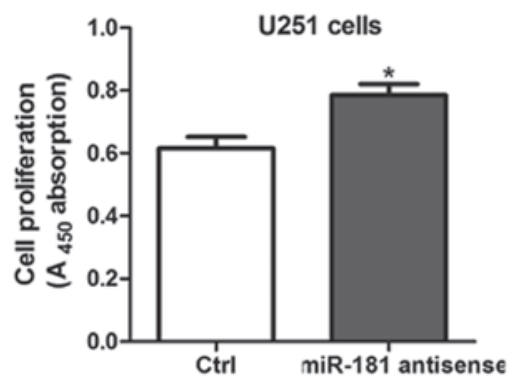

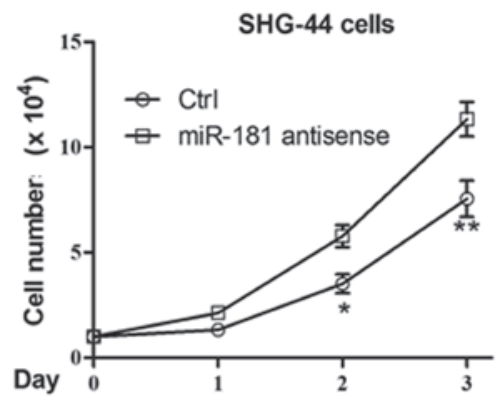

D

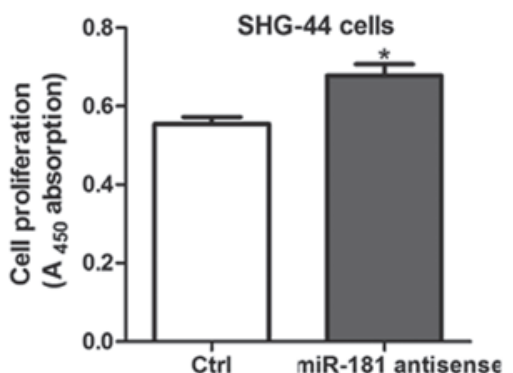

Figure 3. Antisense miR-181 promotes the proliferation of glioma cells. The growth curve of (A) U251 and (B) SHG-44 cells following antisense miR-181 transfection compared with the Ctrl. The cell proliferative potential (BrdU) was determined in (C) U251 and (D) SHG-44 cells transfected with antisense miR-181 or Ctrl. A450 absorption was assayed following transfection for $24 \mathrm{~h}$. ${ }^{*} \mathrm{P}<0.05$ and ${ }^{* *} \mathrm{P}<0.01$, compared with the Ctrl. Ctrl, negative control; miRNA, microRNA.

A

$\begin{array}{cl}\text { Cyclin B1 } & \text { 5'-CCAGCUAAUUGCUACUAGUGGCAAGAGGAACGGAC-3' } \\ \text { miR-181 } & \text { 3'-CUGUUAUUGCAUAGUACAAAGAUUCUCCUUGUGUA-5' }\end{array}$

B

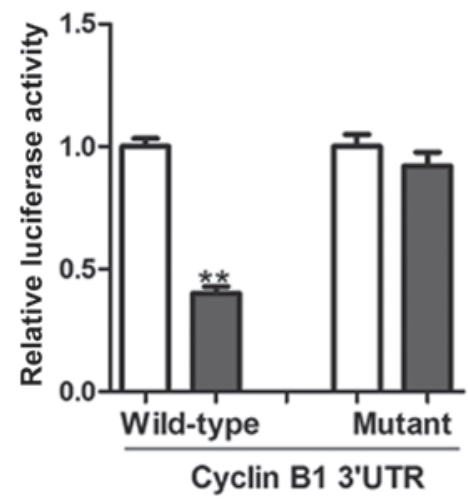

C

U251 Cells

miR-ctrl miR-181 precursor

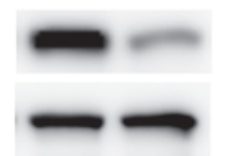

Cyclin B1

GAPDH

E

U251 Cells

Ctrl miR-181 antisense

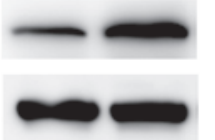

Cyclin B1

GAPDH $\square$ miR-control

miR-181 precursor
D

SHG-44 Cells

miR-ctrl miR-181 precursor

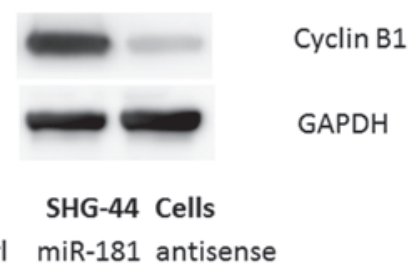

Cyclin B1

GAPDH

Figure 4. miR-181 negatively regulates cyclin B1 expression in glioma cells. (A) Computer prediction of miR-181 binding sites in the 3'UTRs of human cyclin B1 genes. The potential binding site is highlighted in bold. (B) Luciferase reporter assays in U251 cells. Cells were transfected with 100 ng of wild-type

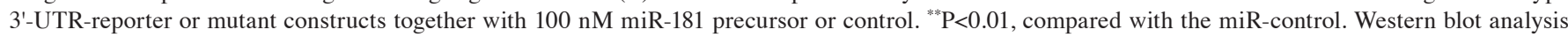
of cyclin B1 in (C) U251 and (D) SHG-44 cells transfected with miR-181 precursor or miR-control. Western blot analysis of cyclin B1 in (E) U251 and (F) SHG-44 cells transfected with antisense miR-181 or negative control. miRNA, microRNA; UTR, untranslated region. 
controls glioma proliferation and invasion by regulating Wnt $/ \beta$-catenin signaling via Nemo-like kinase (16). In addition, miR-200b targets CREB1 and suppresses cell growth in human malignant glioma (17). Furthermore, the downregulation of miR-383 promotes glioma cell invasion by targeting insulin-like growth factor 1 receptor (18). By contrast, miR-107 inhibits U87 glioma stem cell growth and invasion by modulating Notch2 expression $(19,20)$. Therefore, miRNA expression appears to have a key role in regulating cellular processes in glioma, which requires further investigation in the future.

In conclusion, the key finding of the present study is that miR-181 is able to promote the proliferation of glioma cell lines by targeting cyclin B1. This data indicates that miR-181 has an essential role in the regulation of glioma cell proliferation and may function as a tumor suppressor.

\section{References}

1. Wang Y and Jiang T: Understanding high grade glioma: molecular mechanism, therapy and comprehensive management. Cancer Lett 331: 139-146, 2013.

2. Chaudhry NS, Shah AH, Ferraro N, Snelling BM, Bregy A, Madhavan K and Komotar RJ: Predictors of long-term survival in patients with glioblastoma multiforme: advancements from the last quarter century. Cancer Invest 31: 287-308, 2013.

3. Hirst TC, Vesterinen HM, Sena ES, Egan KJ, Macleod MR and Whittle IR: Systematic review and meta-analysis of temozolomide in animal models of glioma: was clinical efficacy predicted? $\mathrm{Br}$ J Cancer 108: 64-71, 2013.

4. Marsh JC, Goldfarb J, Shafman TD and Diaz AZ: Current status of immunotherapy and gene therapy for high-grade gliomas. Cancer Control 20: 43-48, 2013.

5. Pritchard CC, Cheng HH and Tewari M: MicroRNA profiling: approaches and considerations. Nat Rev Genet 13: 358-369, 2012.

6. Kasinski AL and Slack FJ: Epigenetics and genetics. MicroRNAs en route to the clinic: progress in validating and targeting microRNAs for cancer therapy. Nat Rev Cancer 11: 849-864, 2011.

7. Naguibneva I, Ameyar-Zazoua M, Polesskaya A, Ait-Si-Ali S, Groisman R, Souidi M, Cuvellier S and Harel-Bellan A: The microRNA miR-181 targets the homeobox protein Hox-A11 during mammalian myoblast differentiation. Nat Cell Biol 8: 278-284, 2006
8. Pekarsky Y, Santanam U, Cimmino A, Palamarchuk A, Efanov A, Maximov V, Volinia S, Alder H, Liu CG, Rassenti L, Calin GA, Hagan JP, Kipps T and Croce CM: Tcl1 expression in chronic lymphocytic leukemia is regulated by miR-29 and miR-181. Cancer Res 66: 11590-11593, 2006.

9. Neel JC and Lebrun JJ: Activin and TGF $\beta$ regulate expression of the microRNA-181 family to promote cell migration and invasion in breast cancer cells. Cell Signal 25: 1556-1566, 2013.

10. Kim CH, Kim HK, Rettig RL, Kim J, Lee ET, Aprelikova O, Choi IJ, Munroe DJ and Green JE: miRNA signature associated with outcome of gastric cancer patients following chemotherapy. BMC Med Genomics 4: 79, 2011.

11. Ji J, Yamashita T, Budhu A, Forgues M, Jia HL, Li C, Deng C, Wauthier E, Reid LM, Ye QH, Qin LX, Yang W, Wang HY, Tang ZY, Croce CM and Wang XW: Identification of microRNA-181 by genome-wide screening as a critical player in EpCAM-positive hepatic cancer stem cells. Hepatology 50: 472-480, 2009.

12. Wang B, Hsu SH, Majumder S, Kutay H, Huang W, Jacob ST and Ghoshal K: TGFbeta-mediated upregulation of hepatic miR-181b promotes hepatocarcinogenesis by targeting TIMP3. Oncogene 9: 1787-1797, 2010.

13. Chen G, Zhu W, Shi D, Lv L, Zhang C, Liu P and Hu W: MicroRNA-181a sensitizes human malignant glioma U87MG cells to radiation by targeting Bcl-2. Oncol Rep 23: 997-1003, 2010.

14. Goodenberger ML and Jenkins RB: Genetics of adult glioma. Cancer Genet 205: 613-621, 2012.

15. Odjélé A, Charest D and Morin P Jr: miRNAs as important drivers of glioblastomas: a no-brainer? Cancer Biomark 11: 245-252, 2012

16. Wang K, Wang X, Zou J, Zhang A, Wan Y, Pu P, Song Z, Qian C, Chen Y, Yang S and Wang Y: miR-92b controls glioma proliferation and invasion through regulating Wnt/beta-catenin signaling via Nemo-like kinase. Neuro Oncol 15: 578-588, 2013.

17. Peng B, Hu S, Jun Q, Luo D, Zhang X, Zhao H and Li D: MicroRNA-200b targets CREB1 and suppresses cell growth in human malignant glioma. Mol Cell Biochem 379: 51-58, 2013.

18. He Z, Cen D, Luo X, Li D, Li P, Liang L and Meng Z: Downregulation of miR-383 promotes glioma cell invasion by targeting insulin-like growth factor 1 receptor. Med Oncol 30: $557,2013$.

19. Chen L, Chen XR, Zhang R, Li P, Liu Y, Yan K and Jiang XD: MicroRNA-107 inhibits glioma cell migration and invasion by modulating Notch2 expression. J Neurooncol 112: 59-66, 2013.

20. Chen L, Chen XR, Chen FF, Liu Y, Li P, Zhang R, Yan K, Yi YJ, Xu ZM and Jiang XD: MicroRNA-107 inhibits U87 glioma stem cells growth and invasion. Cell Mol Neurobiol 33: 651-657, 2013. 